\title{
Conf-720670--3
}

\section{Information on Grain Boundary Structure in Nanocrystalline Pd and Cr from Diffraction Studies*}

\author{
J. A. Eastman, M. R. Fitzsimmons**, A. C. Lawson**, R. A. Robinson**, \\ L. J. Thompson, and J. Satti \\ Materials Science Division \\ Argonne National Laboratory \\ Argonne, IL 60439 \\ 8 \\ $A N / C P-76522$ \\ DE92 017094
}

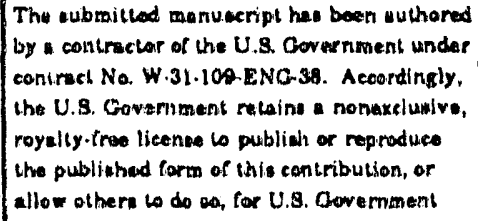

\section{DISCLAIMER}

\begin{abstract}
This report was prepared as an account of work sponsored by an agency of the United Stares Government. Neither the United States Government nor any agency thereof, nor any of their employees, makes any warranty, express or implied, or assumes any legal liability or responsibility for the accuracy', compleieness, or usefulness of any information, apparatus, product, of process disclosed, or represents that its use would not infringe privately owned rights. Reference herein to any specific commercial product, process, or service by trade name, tradermark, manufacturer, of otherwise does not recessarily constitute or imply its endorsement, recommendation, or favoring by the United States Government or any agency theroof. The views and opinions of authors expressed berein do not necessarily state or reflect those of the United States Governmeit or any agency thereol.
\end{abstract}

INVITED paper submitted to the 6th International Conference of Intergranular and Interphase Boundaries in Materials (iib92), Thessaloniki, Greece, June 21-26, 1992.

*Work supported by the U. S. Department of Energy, BES.Materials Sciences, under Contracts W-31-109-Eng-38 and W-7405-Eng-36.

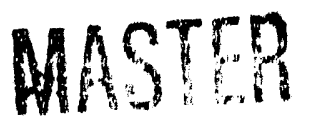<smiles>CC1(C)CCCC1CC1CC1</smiles> 


\title{
Information on Grain Boundary Structure in Nanocrystalline Pd and Cr from Diffraction Studies
}

\author{
J. A. Eastman, M. R. Fitzsimmons", A. C. Lawson"*, R. A. Robinson", \\ L. J. Thompson, and J. Satti \\ Materials Science Division \\ Argonne National Laboratory \\ Argonne, IL. 60439 USA \\ "Los Alarnos National Laboratory \\ LANSCE, Los Alamos, NM 87545 USA
}

Key Words: nanocrystalline metals, $\mathrm{Pd}, \mathrm{Cr}$, grain boundary structure, $\mathrm{X}$-ray diffraction, neutron diffraction

\section{ABSTRACT}

Comparisons of diffraction data from nanocrystalline and coarse-grained Pd samples indicate that grain boundaries in nanocrystalline Pd are reasonably ordered. Lattice parameter measurements show no significant grain boundary excess volume in nanocrystalline $\mathrm{Pd}$, and therefore the reduced density typically observed in nanocrystalline Pd samples must be due to porosity. The lack of any significant change in both thermal expansion coefficients and average atomic vibrational amplitudes may indicate that rigidbody motions normal to grain boundaries in nanocrystalline Pd are severely constrained. Nanocrystalline $\mathrm{Cr}$ shows many similarities in behavior with nanocrystalline Pd, except that significantly more rion-peak intensity is observed from a nanocrystalline $\mathrm{Cr}$ sample than from a coarse-grained $\mathrm{Cr}$ sample. Impurities may account for this behavior.

\section{INTRODUCTION}

Nanocrystalline materials have been shown to possess a variety of interesting altered properties compared to conventional coarse-grained materials (for recent reviews see $[1,2]$ ). The fraction of atoms located in close proximity to grain boundaries is quite high in nanocrystalline materials (for example $~ 50 \%$ of the atoms in a $5 \mathrm{~nm}$ grain-sized material are located within $0.5 \mathrm{~nm}$ of one or more grain boundaries), and thus grain boundaries are expected to have an important influence on the properties of nanocrystalline materials. In order to understand and control any altered properties, characterizing and understanding the structure of grain boundaries in nanocrystalline materials is necessary. 
Previous investigators employing $X$-ray diffraction [3] and EXAFS [4] techniques proposed that the interfaces in nanocrystalline materials represent a new class of solid-state structure lacking both short- and long-range order. Many subsequent studies have attributed changes in properties to the presence of highly disordered boundary structures in nanocrystalline materials. A more recent HREM study [5] disputed the suggestion that the grain boundaries in nanocrystalline $P d$ are highly disordered and concluded that the boundaries in nanocrystalline materials do not differ fundamentally from those in coarsergrained materials. In the present paper it will be shown that the results of diffraction studies of the thermal properties of nanocrystalline Pd $[6,7]$, and the magnetic properties of nanocrystalline $\mathrm{Cr}[8]$ can be used to provide information on the structure of grain boundaries in these materials.

\section{EXPERIMENTAL TECHNIQUES}

Nanocrystalline samples were prepared by evaporating high-purity $\mathrm{Pd}$ or $\mathrm{Cr}$ in $500 \mathrm{~Pa}$ of $99.9999 \%$ pure helium to condense ultrafine particles, and then collecting and consolidating these particles at room temperature under vacuum conditions following the procedures first suggested by Gleiter [9]. The median grain-sizes measured using transmission electron microscopy were $8.3 \mathrm{~nm}$ for nanocrystalline $P d$ and $11 \mathrm{~nm}$ for nanocrystalline $\mathrm{Cr}$. The nanocrystalline $\mathrm{Pd}$ sample was studied by $\mathrm{X}$-ray diffraction, and had an estimated density of $80 \pm 10 \%$ of the bulk $\left(12.02 \mathrm{gm} / \mathrm{cm}^{3}\right)$. Nanocrystalline $\mathrm{Cr}$ samples were examined by both $X$-ray and neutron diffraction, and had estimated densities of $52 \pm$ $10 \%$ of bulk $\operatorname{Cr}\left(7.19 \mathrm{gm} / \mathrm{cm}^{3}\right)$. An as-purchased high-purity commercial foil was used as the coarse-grained $\mathrm{Pd}$ reference sample, while the coarse-grained $\mathrm{Cr}$ reference samples were commercial chips for the neutron diffraction studies, and a foil for the $X$-ray diffraction study. The nanocrystalline $\mathrm{Cr}$ samples were analyzed for $\mathrm{C}, \mathrm{H}$, and $\mathrm{N}$ contents using a LECO-Corporation Model $900 \mathrm{CHN}$ analyzer operated with a furnace temperature of $1000^{\circ} \mathrm{C}$.

The $\mathrm{Pd} X$-ray data were acquired in reflection mode over the temperature range 16 $300 \mathrm{~K}$ using beamline $\times 7 \mathrm{~A}$ at the National Synchrotron Light Source, Brookhaven National Laboratory. The $\mathrm{Cr}$ neutron diffraction data were obtained over approximately the same temperature range at the Los Alamos Neutron Scattering Center using the Hign Intensity Powder Diffractometer. Room temperature Cr X-ray scans were taken at Argonne National

Laboratory using a rotating anode source. Kinematical diffraction theory was used to relate the measured intensity as a function of scattering vector, $\tau=4 \pi \sin \theta / \lambda$, and temperature, to the diffraction process from the samples. For the $X$-ray data, chi-squared minimization datafitting techniques [10] were used to derive information about the shapes, integrated intensities and positions of intensity peaks, as well as the form and magnitude of non-peak 
(diffuse) intensity. Further details are found in [7]. The neutron data were analyzed using the Generalized Structure Analysis System (GSAS) [11].

\section{RESULTS AND DISCUSSION}

Figure 1 shows $X$-ray data obtained at $300 \mathrm{~K}$ for nanocrystalline (points), and coarsegraired (solid line) Pd samples normalized to the same number of incident beam counts. The raw data was corrected for the Lorentz, polarization, and atomic scattering factors, but was not modified to account for the Debye-Waller factor. Increased peak broadening in the nanocrystalline sample is readily apparent. Dashed lines representing the remaining intensity after removing the fitted intensity peaks overlap for the two samples. Raw X-ray data collected at room temperature from nanocrystalline (points) and coarse-grained (solid line) $\mathrm{Cr}$ samples are shown in figure 2. These data were normalized to a constant counttime. The non-peak intensity in figure 2 is shown as a dashed line for the nanocrystalline $\mathrm{Cr}$ sample, and as a solid line for the coarse-grained $\mathrm{Cr}$ sample. Over the measured $\tau$-range, the integrated non-peak intensity from the nanocrystalline $\mathrm{Cr}$ sample was observed to be 2.1 times that from the coarse-grained $\mathrm{Cr}$ sample. A similar enhancement in diffuse scattering from nanocrystalline $\mathrm{Cr}$ was also observed using neutron diffraction. In addition to the intensity peaks from the expected body-centered cubic phase of $\mathrm{Cr}$, additional intensity peaks (arrowed) were identified as coming from a second cubic phase of $\mathrm{Cr}$ that occurs when $\mathrm{Cr}$ is evaporated in an inert-gas environment [12]. From relative intensity comparisons, it was estimated that this phase comprised approximately 2 atomic $\%$ of the nanocrystalline $\mathrm{Cr}$ sample. Other weak intensity peaks from unidentified phases were observed from both samples.

Fig. 1" Corrected X-ray data from nanocrystalline (points) and coarsegrained (solid line) Pd samples. The non-peak portions of the intensities from both samples overlap and are shown as dashed lines. The intensities are plotted on a logarithmic scale versus scattering vector magnitude, $\tau=4 \pi \sin \theta / \lambda$.

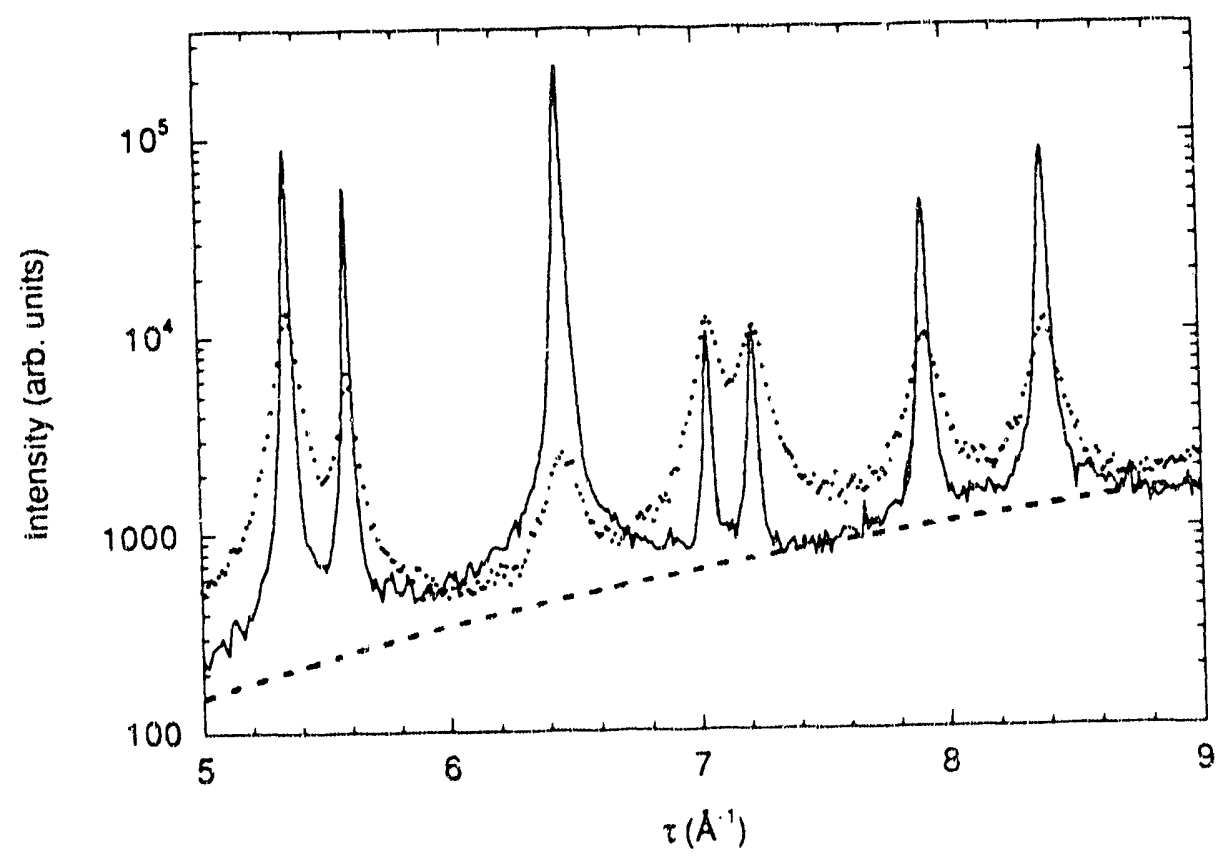


Fig. 2 Raw X-ray data from nanocrystalline (points) and coarsegrained (solid line) $\mathrm{Cr}$ samples. The non-peak portions of the intensities are shown as a dashed line for nanocrystalline $\mathrm{Cr}$, and as a solid line for coarse-grained $\mathrm{Cr}$.

Arrows indicate weak intensity peaks from a non-bcc Cr phase [12]. Additional weak unidentified peaks are

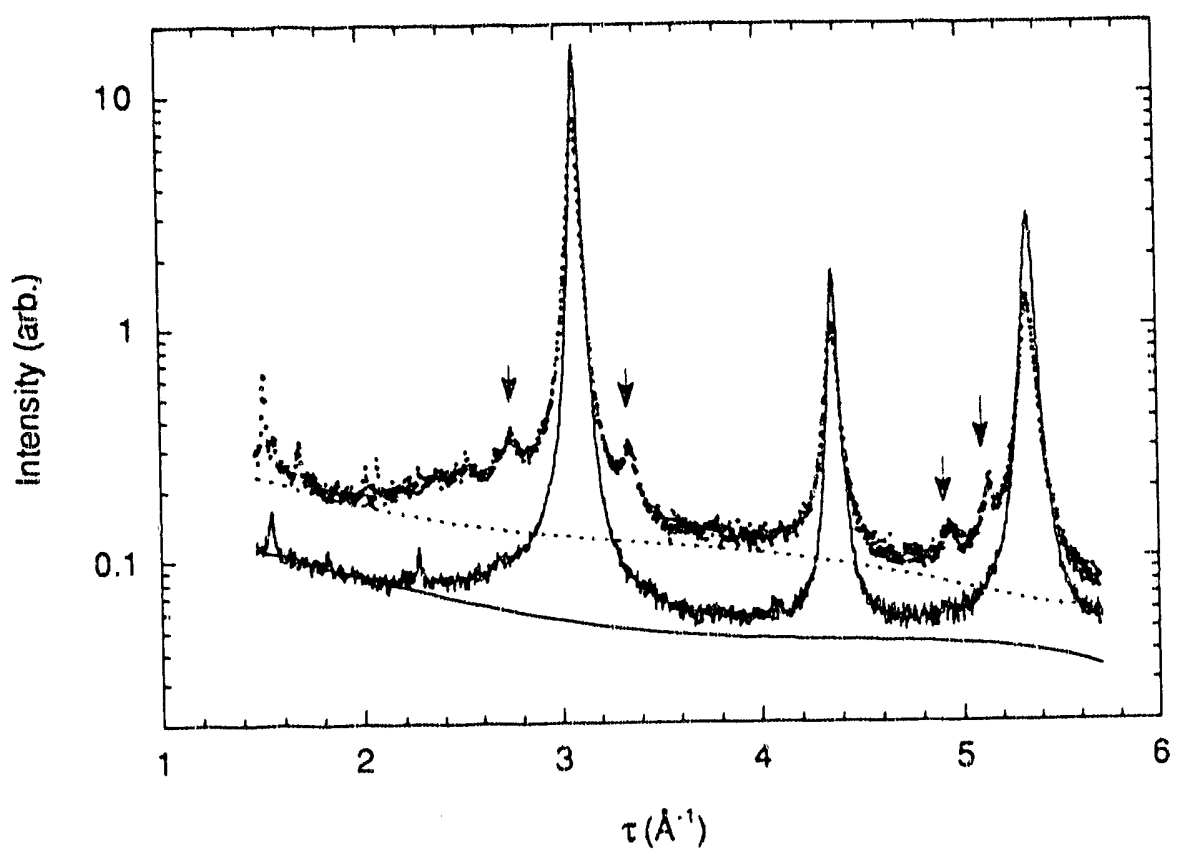
seen for both samples.

Zhu et al. [3] compared $X$-ray data from a nanocrystallirie Fe sample with calculated data from a model in which the nature and magnitude of atomic relaxations in nanocrystalline boundary regions could be controlled. They observed approximately a factor-of-two larger diffuse background component from their nanocrystalline sample than would be expected in the absence of any grain boundary relaxations. Good agreement between calculated and observed diffraction scans required assuming the atoms in a $1 \mathrm{~nm}$ wide boundary region relaxed in random directions by up to $50 \%$ of a nearest-neighbor distance. Since in the present case a comparison of diffuse intensities from nanocrystalline and coarse-grained Pd samples shows no grain-size dependence, this precludes the possibility that a highly-disordered grain boundary phase comprising a significant volume fraction of the sample could be present in nanocrystalline $\mathrm{Pd}$, as had been proposed for nanocrystalline $\mathrm{Fe}$. A large increase in diffuse intensity was observed for nanocrystalline $\mathrm{Cr}$ compared to coarse-grained $\mathrm{Cr}$ (figure 2), such as was predicted for $\mathrm{Fe}$ [3]. While this could suggest that grain boundaries in nanocrystalline face-centered materials like $\mathrm{Pd}$ have fundamentally different structures than those in nanocrystalline body-centered cubic materials such as $\mathrm{Fe}$ and $\mathrm{Cr}$, it is more likely that the additional diffuse scattering from the nanocrystalline $\mathrm{Cr}$ sample was due to the presence of substantial impurity levels in the material. A chemical analysis revealed that the nanocrystalline $\mathrm{Cr}$ contained 0.35 weight $\%$ $\mathrm{H}, 0.97$ weight $\% \mathrm{C}$, and .19 weight $\% \mathrm{~N}$, with an estimated uncertainty of 0.1 weight $\%$. Oxygen analysis of the $\mathrm{Cr}$ samples has not yet been completed. Additional experiments 
with uncontaminated samples are required in order to determine whether the increased diffuse intensity from reactive body-centered cubic materials is due to an intrinsic disordered grain boundary structure.

Since there is no significant difference in diffuse scattered intensity for nanocrystalline and coarse-grained Pd samples, it is possible in this case to estimate some grain boundary structural parameters for this material from diffraction peak intensities, locations, and breadths. Mean-squared atomic displacements can be derived from measurements of the Debye-Waller parameter, and can be separated into thermal and static components. These results are shown in Figure 3 for Pd. By examining the temperature dependence of the displacements, it can be seen that the atomic vibrational amplitudes are the same in nanocrystalline and coarse-grained $\mathrm{Pd}$, but there is an increased static displacement component in the nanocrystalline Pd sample. At room temperature, an atom in the nanocrystalline Pd sample is displaced from a lattice site by an average of $3.1 \% \pm 0.1 \%$ of the bulk nearest-neighbor distance, compared to values of $2.3-2.7 \%$ of this distance for coarse-grained material. It is not possible to distinguish whether these increased static displacements in the nanocrystalline material are uniformly distributed or localized at grain boundaries, since the $X$-ray technique used in this experiment yields only information averaged from the entire sample. Similar results, showing an increase in static displacements, but no change in thermal displacements, were obtained when comparing neutron diffraction data for nanocrystalline and coarse-grained $\mathrm{Cr}$ samples. The differences in observed diffuse scattering from nanocrystalline and coarse-grained $\mathrm{Cr}$ samples indicate that a significant portion of the nanocrystalline $\mathrm{Cr}$ sample does not scatter into the observed intensity peaks. Since it is possible that the grain boundary regions in the nanocrystalline $\mathrm{Cr}$

Fig. 3 Mean-squared atomic displacements versus temperature for nanocrystalline $\mathrm{Pd}(\mathrm{O}-$ [7], -- [6]), and coarsegrained Pd ( $\Delta$-- [13], $\mathbf{A}$-$[14],+\cdots[15])$. Linear fits are provided as a guide to the eye. From [7].

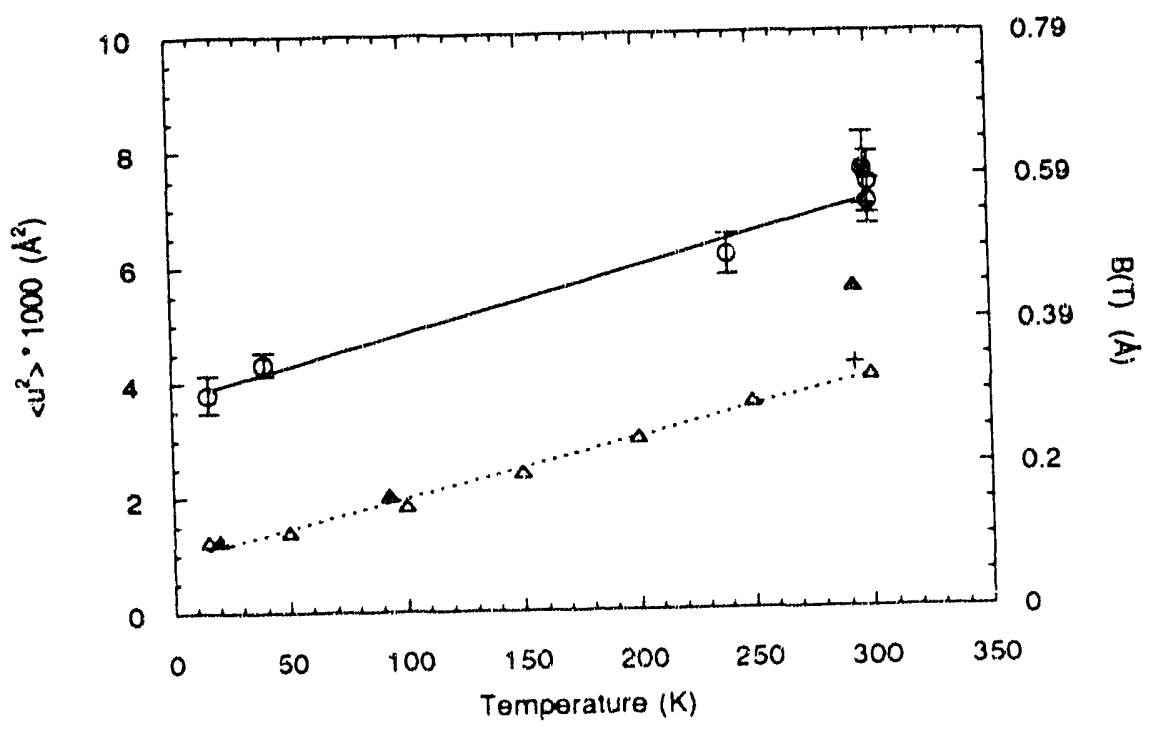


sample were contaminated to the extent that they had an amorphous structure that produced significant diffuse scattering with no contribution to the intensity peaks, this could indicate that the increased static displacements in nanocrystalline Pd are uniformly distributed throughout the grains due to the powder processing, rather than being due to grain boundary relaxations. The same conclusion was reached uy comparing results from the literature for unconsolidated nanometer-scale powders with consolidated material of similar grain size [7].

Lattice parameter comparisons for nanocrystalline and coarse-grained Pd are shown in figure 4. It can be seen from this figure that the total thermal expansion from $16-300 \mathrm{~K}$ of nanocrystalline and coarse-grained $\mathrm{Pd}$ are identical to within $3 \%$, in contradiction to earlier reports of factor 2-3 increases in the thermal expansion coefficient of nanocrystalline materials [1]. There was also no significant difference in the thermal expansion of the crystalline portions of nanocrystallire and coarse-grained $\mathrm{Cr}$ samples. The possibility of differences in the total thermal expansion (including the regions of the nanocrystalline sample that do not contribute to the intensity peaks) can not be ruled out in the case of $\mathrm{Cr}$ though, since different diffuse scattering components were observed for nanocrystalline and coarse-grained $\mathrm{Cr}$, and it is possible that a significant volume of the $\mathrm{Cr}$ sample did not contribute to the intensity peaks used to determine the lattice parameter.

Since the grain boundary regions in nanocrystalline Pd must be scattering into the observed intensity peaks, in this case lattice parameter comparisons can also be used to estimate the grain boundary excess volume, $\Delta \mathrm{V}$, which is defined as:

$$
\Delta V=\left(V_{\text {gb }} V_{\text {bulk }}\right)-1
$$

where $V_{g b}$ and $V_{\text {bulk }}$ are the grain boundary and bulk volumes, respectively for equal masses of material. Defining $V=a_{0}{ }^{3}$, where $a_{0}$ is the lattice parameter, it follows that:

$$
a_{0}(g b)^{3}=(\Delta V+1)^{*} a_{0}(b u l k)^{3}
$$

Defining the measured lattice parameter of a nanocrystalline material, ao (nanocrystalline), as the weighted average of grain boundary and bulk components:

$$
a_{0}(\text { nanocrystalline })=G B F * a_{0}(g b)+(1-G B F) * a_{0}(b u l k)
$$

where GBF is the grain boundary volume fraction in the material, the following relationship between grain boundary excess volume and observed lattice parameters for coarse-grained 
Fig. 4 Lattice parameter as a function of temperature for nanocrystalline (o), and coarse-grained $(\bullet)$ Pd.

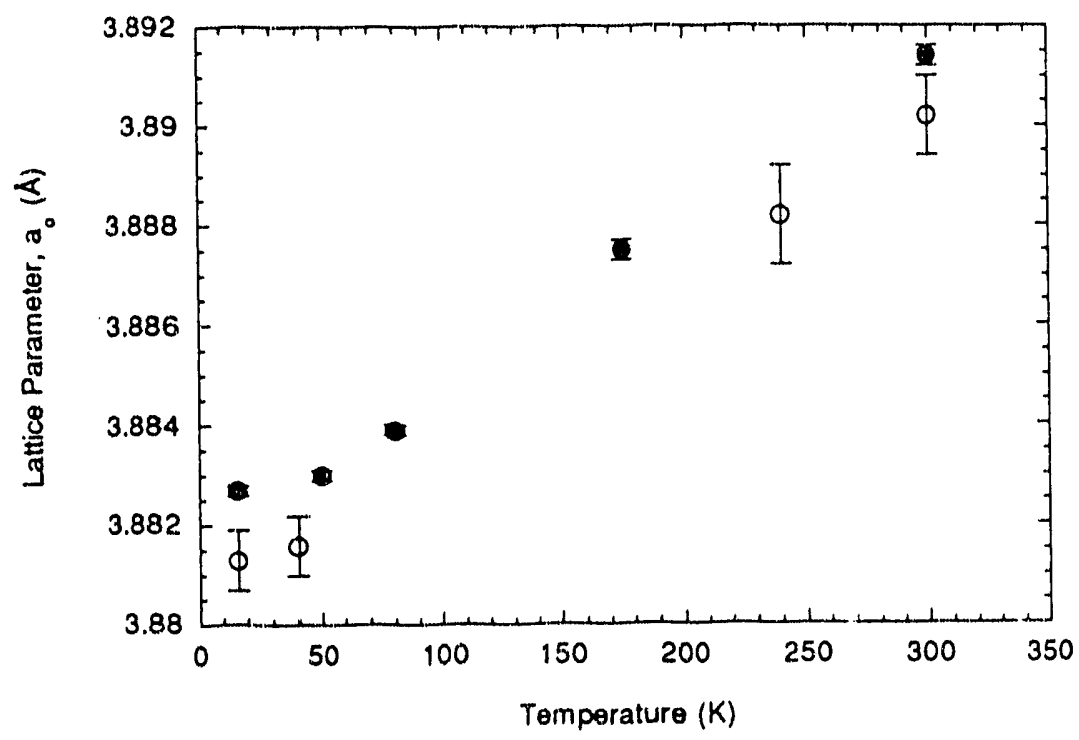

(bulk) and nanocrystalline materials as a function of grain boundary volume fraction is obtained:

$$
a_{0}(\text { nanocrystalline }) / a_{0}(\text { bulk })=(G B F)^{*}(\Delta V+1)^{1 / 3}+(1-G B F)
$$

Recently it has been suggested that observations of reduced density imply that the grain boundaries in nanocrystalline Pd exhibit a large excess volume of $30 \%$ [16]. An $8 \mathrm{~nm}$ grain size and $1 \mathrm{~nm}$ boundary width would correspond to a $33 \%$ grain boundary volume fraction in the absence of porosity in the material. From figure 5, which is obtained from the analysis of equation 4 , it can be seen that this would result in approximately a $3 \%$ increase in lattice parameter in nanocrystalline Pd compared to coarse-grained Pd. The actual lattice parameters for nanocrystalline $\mathrm{Pd}$ with an average grain size of $\sim 8 \mathrm{~nm}$ and coarse-grained Pd differ by less than $0.04 \%$ at both 16 and $300 \mathrm{~K}$ (and the lattice parameter of nanocrystalline $\mathrm{Pd}$ is lower than that of coarse-grained $\mathrm{Pd}$ ). Even if porosity reduces the grain boundary volume fraction to $10 \%$ in the nanocrystalline Pd sample, the volume difference between the bulk and grain boundary regions derived from equation 4 would be only approximately $0.1 \%$ for the observed lattice parameters. From this analysis it is clear that grain boundary regions in nanocrystalline $P d$ do not exhibit a large excess volume, and therefore the reduced density typically observed in nanocrystalline Pd is due to porosity.

The present results indicate that the structure of grain boundaries in nanocrystalline $\mathrm{Pd}$ differs significantly from that of special geometry Au bicrystal boundaries. Fitzsimmons et al. [17] found that the thermal expansion normal to the $A u \Sigma=13$ [001] twist boundary plane is three times larger than the bulk value. The magnitude of this expansion would also be easily observed as a lattice parameter shift in an $8 \mathrm{~nm}$ grain-sized sample. The fact that 
Fig. 5 Grain boundary excess volume, $\Delta \mathrm{V}=\left(\mathrm{V}_{\mathrm{gb}} / N_{\text {bulk }}\right)-1$, versus the ratio of the nanocrystalline and bulk (coarse-grained) lattice parameters. GBF represents the fraction of the nanocrystalline material located in grain boundary regions.

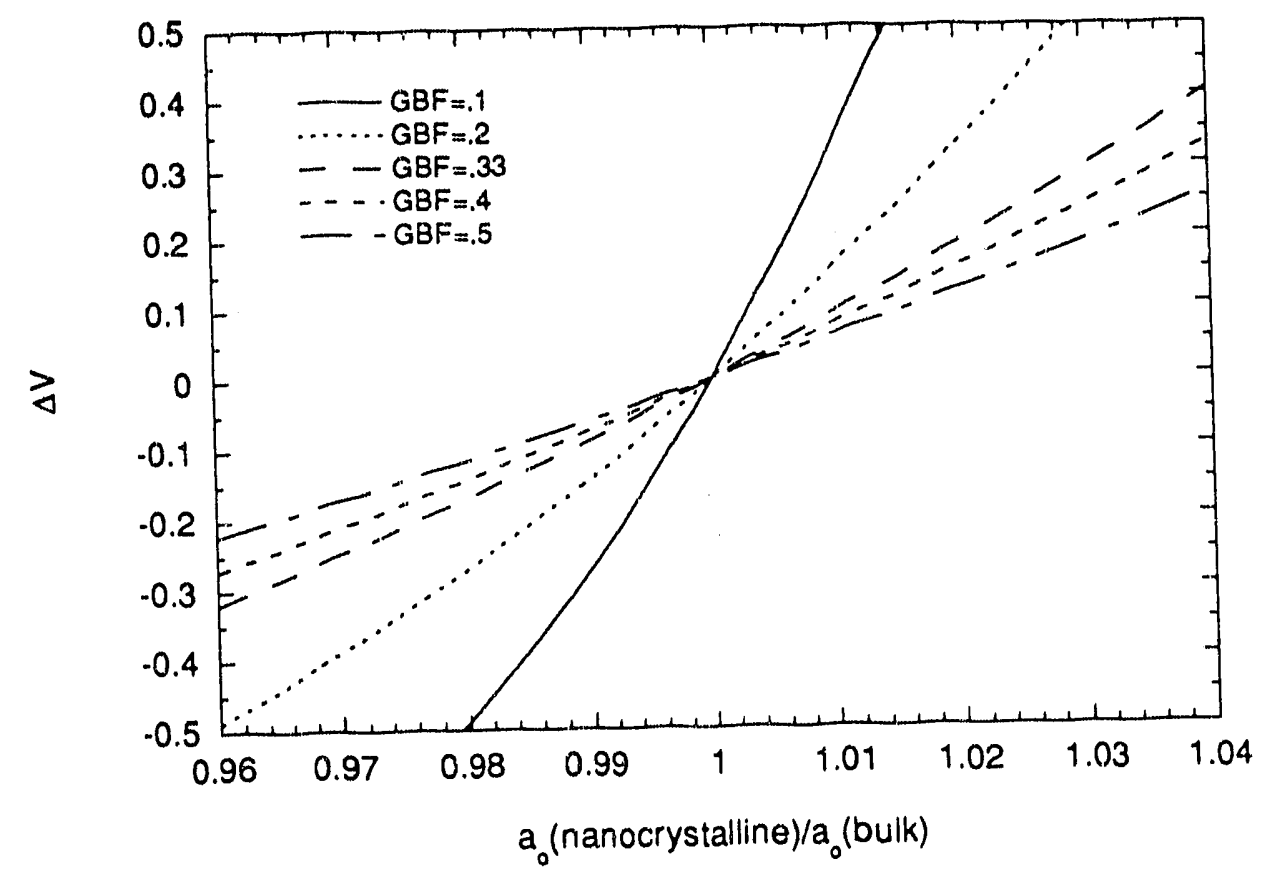

an increase in the thermal component of the grain boundary atom mean-square displacements compared to the bulk displacements was observed in the Au bicrystal work, while no differences in the thermal component of the Debye-Waller parameter were detected in the present work, is further evidence for differences in behavior for grain boundaries in bicrystals compared to boundaries in nanocrystalline materials. One possible explanation for the difference between the present results and the bicrystal observations is that the expansion of a bicrystal is not constrained by neighboring crystallites, while this constraint is present in nanocrystalline materials. In other words, rigid-body motions normal to the boundary plane in bicrystals do not require cooperative shear motion of other nearby boundaries. Alternatively, the observed differences in behavior could simply indicate that random high angle boundaries in Pd differ significantly in structure and behavior compared to a $\Sigma=13[001]$ twist boundary in Au.

\section{CONCLUSIONS}

The primary conclusions of this study are:

1) Comparisons of non-peak intensities in nanocrystalline and coarse-grained samples indicate that grain boundaries in nanocrystalline $\mathrm{Pd}$ are reasonably ordered.

2) There is no evidence for large grain boundary excess volumes in nanocrystalline Pd. Therefore the reduced densities iypically observed in nanocrystalline $\mathrm{Pd}$ samples are due to porosity. 
3) The lack of any observed increase in thermal expansion coefficient and thermal vibrational amplitudes in nanocrystalline Pd compared to coarse-grained Pd suggest that rigid-body motions normal to grain boundaries in nanocrystalline $\mathrm{Pd}$ are severely constrained compared to those in special geometry bicrystal boundaries.

4) Uncontaminated samples are required before definitive information can be obtained regarding the nature of grain boundary structure in reactive nanocrystalline materials such as $\mathrm{Cr}$.

\section{ACKNOWLEDGMENTS}

This work was supported by the U. S. Department of Energy, BES-DMS, under Contracts W-31-109-Eng-38 and W-7405-Eng-36. The research was carried out in part at the National Synchrotron Light Source, Brookhaven National Laboratory, which is supported by the U. S. Department of Energy, Division of Materials Sciences and Division of Chemical Sciences. The Manuel Lujan Jr., Neutron Scattering Center is a national user facility funded by the U. S. Department of Energy, Office of Basic Energy Science. We thank G. Knapp and M. Beno for their assistance in acquiring the $\mathrm{Cr} X$-Ray data. We acknowledge I. Fox and the Argonne Analytical Chemistry Laboratory for providing the $\mathrm{Cr}$ CHN analyses.

\section{REFERENCES}

1) Gleiter, H., Prog. Mater. Sci., 33, 223 (1989).

2) Andres, R. P., et al., Jn. Mater. Res., 4, 704 (1989).

3) Zhu, X., Birringer, R., Herr, U., and Gleiter, H., Phys. Rev. B, 35, 9085 (1987).

4) Haubold, T., Birringer, R., Lengeler, $B$., and Gleiter, H., Jn. Less-Common Metals, 145, 557 (1988).

5) Thomas, G. J., Siegel, R. W., and Eastman, J. A., Scripta Met., 24, 403 (1990).

6) Fitzsimmons, M. R., Eastman, J. A., Müller-Stach, M., and Wallner, G., Phys. Rev. B, 44, no. 6, 2452 (1991).

7) Eastman, J. A., Fitzsimmons, M. R., and Thompson, L. J., accepted by Phil. Mag. B.

8) Fitzsimmons, M. R., Eastman, J. A., Lawson, A., Robinson, R., and Satti, J., in preparation.

9) Gleiter, H., Deformation of Polycrystals: Mechanisms and Microstructures, edited by N. Hansen et al., Roskilde: Risø National Laboratory, 15 (1981).

10) Press, W. H., Flannery, B. P., Teukolsky, S. A., and Vetterling, W. T., Numerical Recipes, Cambridge, New York: Cambridge University Press (1989).

11) Larson, A. C., and Von Dreele, R. B., LAUR 86-748, Los Alamos National Laboratory, (1966).

12) Kimoto, K. and Nishida, I., Jn. Phys. Soc. Jpn., 22, no. 3, 744 (1967). 
13) Lawson, A., Conant, J. W., Robertson, R., Rohwer, R. K., Young, V. A.Talodtt, C. L., accepted by J. Alloys and Compounds

14) International Tables for $X$-ray Crystallography III, Birmingham: Kynoch Press (1968).

15) Nelin, G., Phys. Stat. Sol. B, 45, 527 (1971).

16) Wagner, M., Phys. Rev. B, 45, no. 2, 635 (1992).

17) Fitzsimmons, M. R., Burkel, E., and Sass, S. L., Phys. Rev. Lett., 61, no. 19, 2237 (1988). 

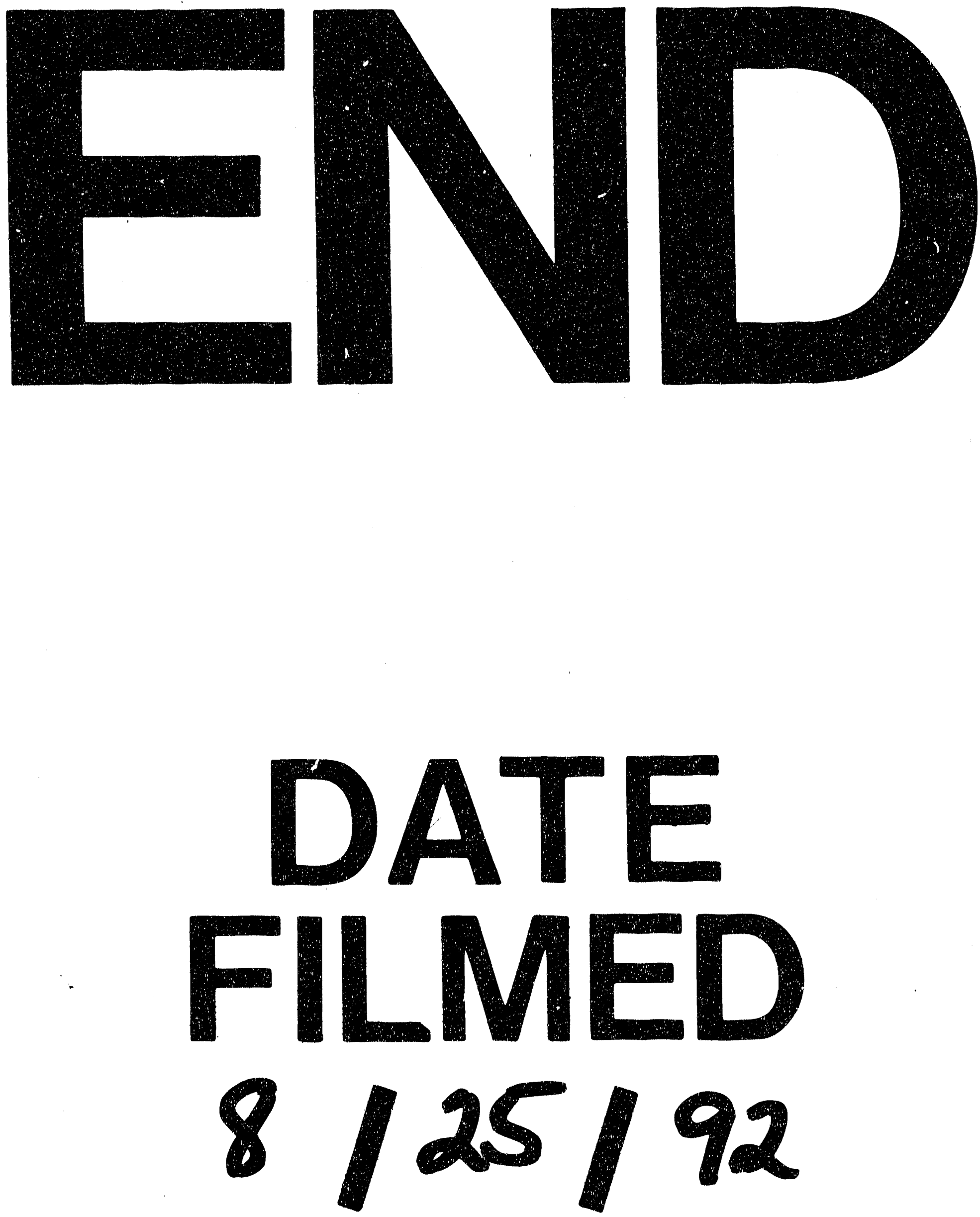